\title{
COMPARATIVE MEASUREMENTS OF HEAD ANGULAR MOVEMENTS USING A CAMERA SYSTEM AND A GYROSCOPE SYSTEM
}

\author{
Vladimir Socha $^{a, *}$, Patrik Kutilek $^{a}$, Ondrej Cakrt $^{b}$, Rudolf Cerny ${ }^{b}$ \\ ${ }^{a}$ Faculty of Biomedical Engineering, Czech Technical University in Prague, Czech Republic \\ ${ }^{b}$ 2nd Faculty of Medicine - Motol University Hospital, Charles University in Prague, Czech Republic \\ * corresponding author: ing.vladimir.socha@gmail.com
}

\begin{abstract}
Assessments of body-segment angular movements are very important in the rehabilitation process. Head angular movements are measured and analyzed for use in studies of stability and posture. However, there is no methodology for assessing angular movements of the head, and it has not been verified whether data measured by fundamentally different MoCap systems will lead to the same results. In this study, we used a camera system and a 3DOF orientation tracker placed on the subject's head, and measured inclination (roll) and flexion (pitch) during quiet stance. The total length and the mean velocity of the traces of the pitch versus roll plots were used to measure and analyze head orientation. Using these methods, we are able to model the distribution of the measured 2D data, and to evaluate stability and posture. The results show that the total lengths and the mean velocities related to the 3DOF orientation tracker do not differ significantly from the total lengths and the mean velocities of traces related to the IR medical camera. We also found that the systems are not interchangeable, and that the same type of system must be used each time. The designed methods can be used for studies not only of head movements but also of movements of other segments of the human body, and can be used to compare other types of MoCap systems, depending on the requirements for a specific rehabilitation examination.
\end{abstract}

KEYWORDS: head orientation, camera system, orientation tracker, pitch versus roll plots.

\section{INTRODUCTION}

The position of the head can be negatively influenced by many diseases of the nervous system [1]. Patients with vestibular deficits often show instability during stance tasks. Making the stance task more difficult by removing visual inputs has been claimed as a means for identifying a vestibular deficit 2, 3. MoCap systems were used for 3-D high-accuracy measurements of human body segments, instead of widely-used force platforms for studying the centre of pressure (CoP). Sensing units (in the case of the inertial system) or markers (in the case of the camera system) were used to measure the pitch, roll and yaw of the segments. The techniques were introduced to quantify the movements of segments in both anterior-posterior and medial-lateral directions during stance [3, 4]. Measurements of head angular movements during stance can detect changes in postural stability [5]. It is well known that the sway of standing patients with a vestibular deficit is mainly side-to-side (in the roll plane), and they fall to the side of the deficit [4. Instability in other directions, such as the fore-aft (pitch) plane, are also observed [6]. Therefore, an assessment of angular movements in the roll plane and the fore-aft plane may yield clearer insights into balance deficits and provide a considerably better diagnostic tool than other more traditional measures of postural instability. This study will therefore use and compare 2D data about the inclination (roll) and flexion (pitch) of a head measured by two fundamentally different MoCap systems - a medical camera with markers, and a tri-axial inertial measurement unit (i.e., a 3DOF orientation tracker). These two fundamentally different systems are basic MoCap systems for studying postural stability in $3 \mathrm{D}$ space. The traditional technique for assessing postural instability is a method based on recordings of the $\mathrm{CoP}$ with the aid of a static force platform [7. This technique aims to compare the practical usefulness of parameters of body sway. These include: mean displacement velocity, total length of the CoP trajectory, movement area, planar deviation, etc. The methods are based on a description of the behavior of two variables in two planes/axes. However, we need to model the distribution of the measured 2D data (pitch, roll), because we use MoCap systems instead of the widely-used force platforms. XY plots of pitch and roll angle can be used for studying the 2 D data $[8,9]$.

\subsection{AIM OF THE WORK}

Using a method based on the total length of the trajectory and the mean displacement velocity of pitch versus roll plots, the head sway measurements recorded during stance tasks can provide useful information for identifying balance deficits and disorders. The total length of the trajectory and the mean displacement velocity of the pitch versus roll plots were used to 


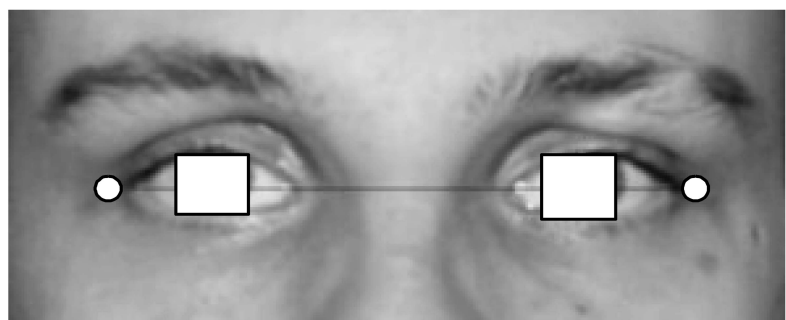

\section{a) Anatomical horizontal}

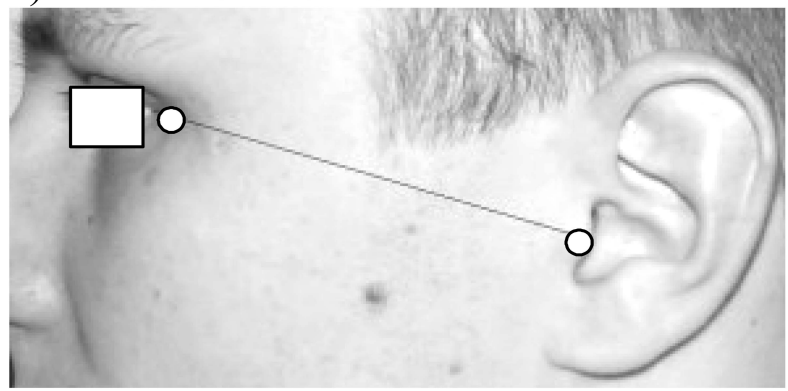

b) Anatomical axis

Figure 1. Anatomical Frankfort horizontal and axis [3].

assess head angular movements. The second question was whether 2D data measured by two fundamentally different MoCap systems will lead to the same results. Two MoCap systems were compared on the basis of the total length of the trajectory and the mean displacement velocity of the pitch versus roll plots.

\section{Methods AND MATERIALS}

\subsection{Subjects}

Ten healthy subjects (control group - CG; aged 22.2 (SD 1.4) years) were recruited from the students at the Czech Technical University in Prague. In brief, head orientation was measured during stance. The tasks involved standing on both legs on a firm surface for 60 seconds with eyes open (EO) and with eyes closed (EC). The subject's feet were positioned next to each other, splayed at $30^{\circ}$, arms always in hanging position 10. Each subject was measured three times with 2-minute intervals between each measurement.

\subsection{Motion CAPTURE EQUiPMEnT}

We used one medical camera system with active markers and one inertial measurement unit. We used the Lukotronic AS 200 camera system (Lutz Mechatronic Technology e.V.). This is a widely-used medical camera system with active markers. The markers are placed in accordance with the Frankfort horizontal, see Fig. 1, for measuring angular movements [3]. Active markers were placed on the following anatomical points: the left and right tragus and the left and right outer eye canthus, Fig. 1. MatLab software (The MathWorks Inc.) was used to identify the angles (pitch, roll, yaw) of the head in 3D space [10. The camera system was calibrated before the experiments, and the origin of the world coordinate system was set

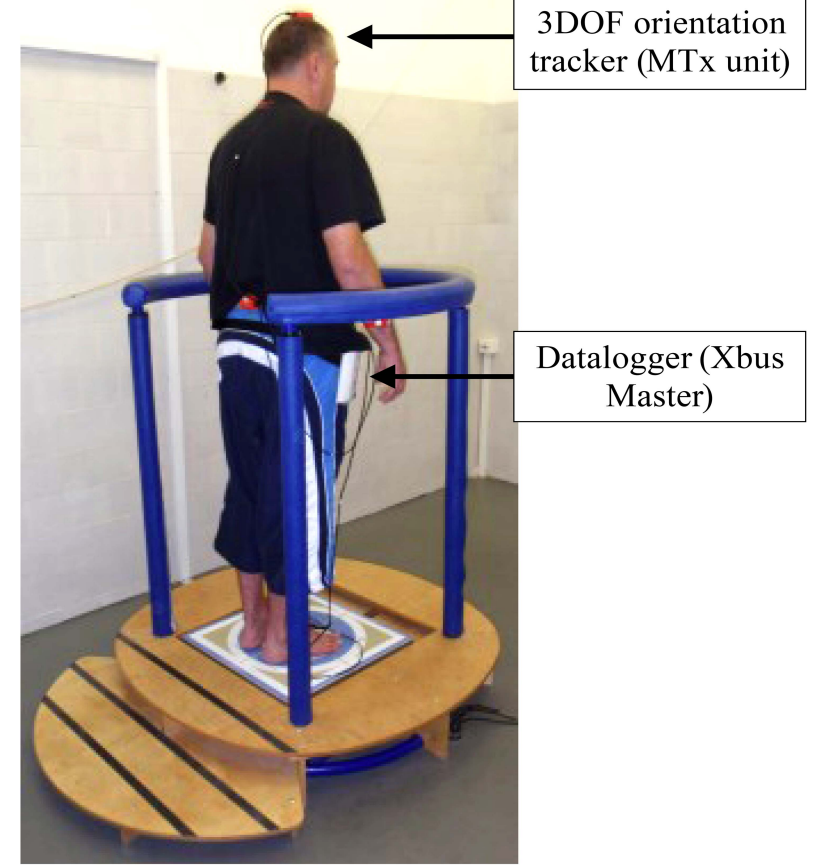

Figure 2. Illustration of the Xsens system with an MTx gyro sensor placed on the patient's head.

up so that the first axis was along the symmetry axis (coinciding with the anterior posterior axis) of the platform on which the subjects stood, and the other two axes were perpendicular to the symmetry axis of the platform. The sample frequency was $100 \mathrm{~Hz}$.

For head sway measurements, the Xbus Master motion capture system was also used. This is a lightweight device that uses MTx units for orientation measurements of segments [12, see Fig. 22 The MTx unit is an accurate $3 \mathrm{DOF}$ tracker. The one MTx unit was placed on the patient's head, in accordance with Raya at al. 13 and Casolo [14, see Fig. 2 The head sway was measured in three planes, and the sample frequency was $100 \mathrm{~Hz}$.

Each simultaneous measurement of one subject with $\mathrm{EO}$ or EC took one minute. It is not necessary to normalize the data, because the standard ranges of the angles are the same for the three planes of the body, and for all adult persons, if the 3DOF orientation tracker and/or markers are placed on the same segment (i.e., points). Using these techniques, we can record and study the head movements.

\subsection{METHOD OF QUANTIFICATION OF POSTURAL STABILITY}

The method used for analyzing head movements is based on the total length of the trajectory and the mean displacement velocity of the pitch versus roll plots [8, 9]. The reason for using this method is that the standardized descriptives, such as the standard deviations of displacement and planar deviation seem to be less useful, because the parameter appears to be independent of the sampling frequency, and is not correlated to any of the trajectory parameters [7] that 


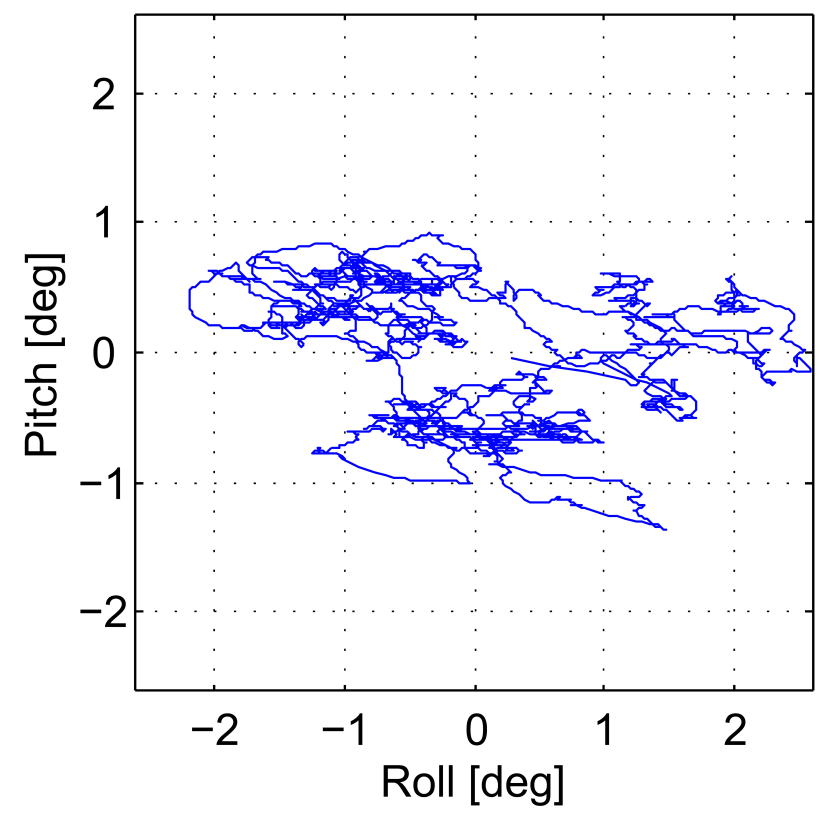

Figure 3. Example of a pitch versus roll plot of a head.

directly reflect the measured movement of the body segment. We may add that the total length of the trajectory of the plot is directly arithmetically related to the mean velocity and the recording time (which was the same in all our recordings), and provides similar information, provided the recording time is standardized. The method of the pitch versus roll plots applied to measured data forms a trajectory, Fig. 3. The total length and the mean velocity is given by formulas described in detail in [7].

\subsection{Statistical ANALYSis}

After calculating the total length of the trajectory and the mean displacement velocity of the pitch versus roll plots of each subject with EO or EC, measured by both systems, a statistical analysis was performed with the use of MatLab software. We calculated the minimum (Min), maximum (Max), median, first quartile (Q1), third quartile (Q3), mean and standard deviation (SD) for the total length, and the mean velocity of the pitch versus roll plots. We used the descriptive statistic to illustrate the relationship between the subjects with EO and EC. The Jarque-Bera test was used to test the normal distribution of all parameters (at a significance level of $5 \%$ ).

The Wilcoxon signed rank test was used to assess the significance of the differences between the total length of the pitch versus roll plots of the measured subjects, with EO and EC, measured by the Lukotronic system and the Xsens system, and to assess the significance of the differences between mean velocity of the pitch versus roll plots of the measured subjects, with EO and EC, measured by the Lukotronic system and the Xsens system. The data about the total length and the mean velocity of the pitch versus roll plots of the subjects measured by the Lukotronic system were compared with data about the total length and the mean velocity of the pitch versus roll plots of the subjects measured by the Xsens system. The significance level was set at $p<0.05$.

\section{Results}

After calculating the total lengths and the mean velocities of the traces of the pitch versus roll plots, a statistical analysis was performed, see Tables 1 and 2 The charts in Figures 4 and 5 show the relationship between the total lengths of the traces of subjects with $\mathrm{EO}$ and $\mathrm{EC}$, and between the mean velocities of traces of subjects with EO and EC.

The Jarque-Bera test returns $h=1$ in two cases and $h=0$ in six cases for measurements of the total length and the mean velocity of the traces of the pitch versus roll plots. Since some data did not have a normal distribution, the Wilcoxon test was used to analyze the data.

\subsection{Total Length of the traces of the PITCH VERSUS ROLL PLOTS}

In the case of the total length of the traces of the pitch versus roll plots of the subjects with EO and EC measured by the Lukotronic system, the results showed no significant difference in the length of the traces $(p=0.44)$. In the case of the total length of the traces of the pitch versus roll plots of the subjects with EO and EC measured by the Xsens system, the results also showed no significant difference in the length of the traces $(p=0.96)$. All calculated p-values were greater than the significance level $(p<0.05)$. Therefore, we do not reject the null hypothesis, and we can state that there is no significant difference between the total length of the traces of the subjects with EO and EC.

Subjects with EO do not demonstrate a significant difference in the length of the traces $(p=0.24)$ measured by the Xsens system and the Lukotronic system. Subjects with EC also do not demonstrate a significant difference in the length of the traces $(p=0.44)$ measured by the Xsens system and the Lukotronic system. All calculated $p$-values were higher than the significance level $(p<0.05)$. There is therefore no significant difference between the data measured by the Lukotronic system and the data measured by the Xsens system. The median of the length of the traces related to the subjects with EO and measured by the Xsens system is 1.35 times larger than the median of the length of the traces related to the subjects with EO and measured by the Lukotronic system. The median of the length of the traces related to the subjects with EC and measured by the Xsens system is 1.28 times larger than the median of the length of the traces related to the subjects with EC and measured by the Lukotronic system. 


\begin{tabular}{lrrrc}
\hline & $\begin{array}{c}\text { Lukotr. } \\
\text { CG EO }\end{array}$ & $\begin{array}{c}\text { Xsens } \\
\text { CG EO }\end{array}$ & $\begin{array}{c}\text { Lukotr. } \\
\text { CG EC }\end{array}$ & $\begin{array}{c}\text { Xsens } \\
\text { CG EC }\end{array}$ \\
\hline Min & 60.54 & 65.18 & 65.76 & 70.38 \\
\hline Max & 175.48 & 278.51 & 237.16 & 490.40 \\
\hline Mean & 115.47 & 156.87 & 132.53 & 186.46 \\
\hline SD & 49.72 & 67.86 & 63.09 & 134.93 \\
\hline Median & 114.29 & 154.52 & 111.11 & 141.81 \\
\hline Q1 & 67.81 & 112.86 & 86.78 & 105.24 \\
\hline Q3 & 158.11 & 190.59 & 182.73 & 221.49 \\
\hline
\end{tabular}

TABle 1. Descriptive statistics of the total length of the traces of the pitch versus roll plots of CG with eyes open (EO) and with eyes closed (EC). All values are in degrees $\left(^{\circ}\right)$.

\begin{tabular}{lrrrr}
\hline & $\begin{array}{c}\text { Lukotr. } \\
\text { CG EO }\end{array}$ & $\begin{array}{c}\text { Xsens } \\
\text { CG EO }\end{array}$ & $\begin{array}{c}\text { Lukotr. } \\
\text { CG EC }\end{array}$ & $\begin{array}{c}\text { Xsens } \\
\text { CG EC }\end{array}$ \\
\hline Min & 1.04 & 1.10 & 0.90 & 1.21 \\
\hline Max & 3.08 & 12.36 & 4.22 & 10.22 \\
\hline Mean & 2.11 & 3.85 & 2.18 & 4.09 \\
\hline SD & 0.82 & 3.60 & 1.15 & 3.39 \\
\hline Median & 2.21 & 2.68 & 1.74 & 2.87 \\
\hline Q1 & 1.43 & 2.00 & 1.45 & 1.76 \\
\hline Q3 & 2.74 & 3.70 & 3.04 & 4.91 \\
\hline
\end{tabular}

TABLE 2. Descriptive statistics of the mean velocity of the traces of the pitch versus roll plots of CG with eyes open (EO) and with eyes closed (EC). All values are in degrees per second $(\%)$.

\subsection{Mean Displacement Velocity of the PITCH VERSUS ROLL PLOTS}

In the case of the mean velocity of the traces of the pitch versus roll plots of the subjects with EO and EC measured by the Lukotronic system, the results showed no significant difference in the mean velocity of the traces $(p=0.98)$. In the case of the velocity of the traces of the pitch versus roll plots of the subjects with EO and EC measured by the Xsens system, the results also showed no significant difference in the velocity of the traces $(p=1.0)$. All calculated $p$-values were greater than the significance level $(p<0.05)$. We therefore do not reject the null hypothesis, and there is no significant difference between the mean velocity of the traces of the subjects with EO and EC.

Subjects with EO show no significant difference in the mean velocity of the traces $(p=0.21)$ measured by the Xsens system and the Lukotronic system. Subjects with EC also showed no significant difference in the velocity of the traces $(p=0.21)$ measured by the Xsens system and the Lukotronic system. All calculated $p$-values were higher than the significance level $(p<0.05)$. There is therefore no significant difference

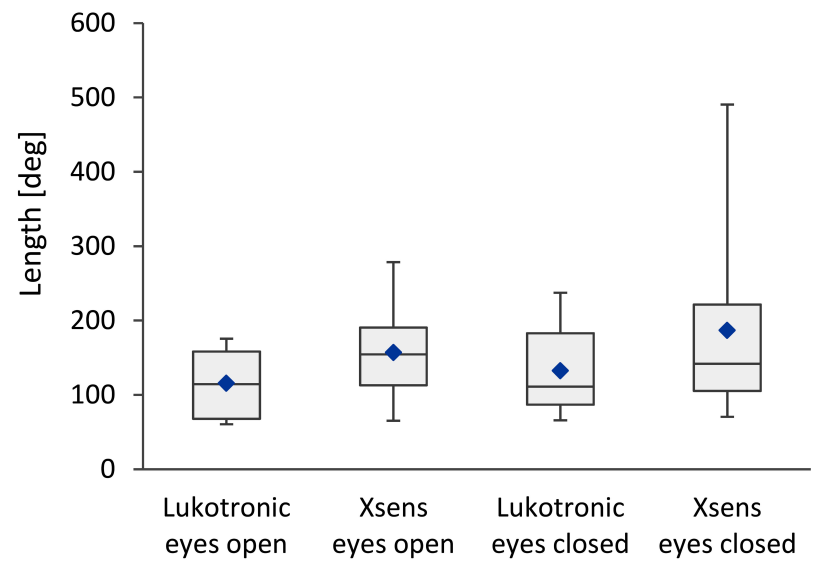

Figure 4. Comparison of the total length of the traces of the pitch versus roll plots of CG with eyes open and with eyes closed. Measured by the Lukotronic and Xsens systems.

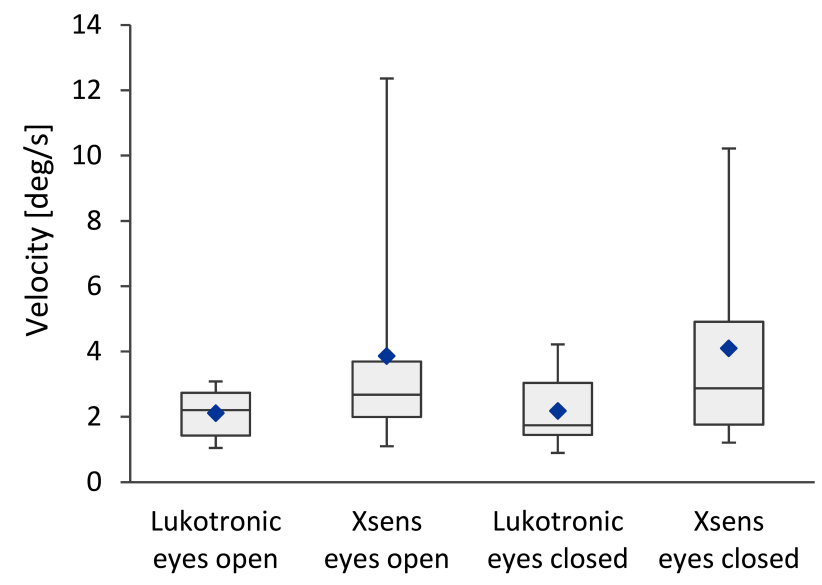

Figure 5. Comparison of the mean velocity of the traces of the pitch versus roll plots of CG with eyes open and with eyes closed. Measured by the Lukotronic and Xsens systems.

between the data measured by the Lukotronic system and the data measured by the Xsens system. The median of the velocity of the traces related to the subjects with EO and measured by the Xsens system is 1.21 times larger than the median of the velocity of the traces related to the subjects with $\mathrm{EO}$ and measured by the Lukotronic system. The median of the velocity of the traces related to the subjects with EC and measured by the Xsens system is 1.65 times larger than the median of the velocity of the traces related to the subjects with EC and measured by the Lukotronic system.

\section{Discussion}

According to the Wilcoxon signed rank test, the results show that the median of the total lengths of the traces related to the subjects measured by the Xsens system is not significantly larger than the median of the total lengths of the traces related to the subjects measured by the Lukotronic system. The 
results also show that the median of the mean velocities related to the subjects measured by the Xsens system is not significantly larger than the median of the mean velocities related to the subjects measured by the Lukotronic system. Generally, however, the medians for the subjects measured by the Xsens system are higher than the medians for the subjects measured by the Lukotronic system. The reason for the different results is gyro sensor drift and skin artifacts, which significantly affect the accuracy. The systems are therefore not interchangeable, and the same type of system must be used each time.

It is also evident that the statistical analysis (Wilcoxon signed rank test) of the subjects with EO and EC showed no significant difference in the total lengths of the traces and/or the mean velocities. Although we had expected some deterioration in the postural stability of the subjects with EC, the results are not conclusive. However, in most cases, the variability (i.e., also the maxima) for subjects with EC was slightly higher than the variability for subjects with EO. There is deterioration in stability and posture, but it is negligible (according to the Wilcoxon signed rank test). A very interesting finding is that, in most cases, the medians for subjects with EC are slightly lower than the medians for subjects with EO. Although the results are slightly different when different MoCap systems are used to measure the inclination and flexion of the head, the MoCap systems identified the same behavior of the subject.

\section{Conclusions}

We have found that the total length of the trajectory and the mean displacement velocity of the pitch versus roll plots based on the data measured by a camera system and/or the total length of the trajectory and the mean displacement velocity based on the data measured by a gyroscope system is suitable for studying stability and posture. The findings have also shown that a single 3DOF orientation tracker placed on the patient's head can replace the complex and more expensive camera system.

The median of the total length of the trajectory is $114.3^{\circ}$ when we use the Lukotronic system and CG with EO, and $111.1^{\circ}$ when we use CG with EC. The median of the total length of the trajectory is $154.5^{\circ}$ when we use the Xsens system and CG with EO, and $141.81^{\circ}$ when we use CG with EC. The median of the mean displacement velocity of the pitch versus roll plots is $2.21^{\circ} / \mathrm{s}$ when we use the Lukotronic system and CG with EO, and $1.74^{\circ} / \mathrm{s}$ for CG with EC. The median of the mean displacement velocity of the pitch versus roll plots is $2.68^{\circ}$ when we use the Xsens system and $\mathrm{CG}$ with $\mathrm{EO}$, and $2.87^{\circ}$ for CG with EC. It should be mentioned that the mean velocity and trajectory length depend significantly on sampling frequency and measuring time [7. In our case, the sampling frequency was $100 \mathrm{~Hz}$ and the measuring time was $60 \mathrm{~s}$.
The concept of mean velocity and trajectory length is known to the biomechanics community, but has not been used before for comparing MoCap systems based on different measurement principles, or for studying head angular movements. The methods can also be used for comparing other types of MoCap systems, and for studying other segments of the human body, depending on the needs of a specific rehabilitation examination. Future work can focus on comparing other systems and movements of segments of the human body.

\section{ACKNOWLEDGEMENTS}

This research has been supported by CTU in Prague projects number SGS 13/091/OHK4/1T/17 and SGS 14/170/OHK4/2T/17.

\section{REFERENCES}

[1] Cerny, R. et al: Head in Space - Noninvasive Measurement of Head Posture. In: "11th Danube Symposium - International Otorhinolaryngological Congress". Bologna: Medimond S.r.l., 2006, p. 39-42.

[2] Ochi, F. et al: Trunk motion analysis in walking using gyro sensors, In. "19th International Conference of the IEEE/EMBS". Chicago: Engineering in Medicine and Biology Society, 1997, p. 1824-1825. ISBN 0-7803-4265-8. DOI:10.1109/IEMBS.1997.757084

[3] Kutilek, P. et al: Methods of measurement and evaluation of eye, head and shoulders position in neurological practice. In: "Advanced topics in neurological disorders". Rijeka: InTech, 2012, p. 25-44. ISBN 978-953-51-0303-5. DOI:10.5772/31378

[4] Allum, J. et al: Trunk sway measures of postural stability during clinical balance tests: effects of a unilateral vestibular deficit. Gait Posture, 14(3), 2001, p. 227-237. DOI:10.1016/S0966-6362(01)00132-1

[5] Hozman, J. et al: Precise Advanced Head Posture Measurement. In: "3rd WSEAS Int. Conf. on Remote Sensing". Athens: WSEAS Press, 2007, p. 18-26. ISBN 978-960-6766-17-6.

[6] Brandt, T., Daroff, R.: The multisensory physiological and pathological vertigo syndromes. Ann. Neurol., 1980, 7(3), p. 195-203. DOI:10.1002/ana.410070302

[7] Raymakers, J.A. et al: The assessment of body sway and the choice of the stability parameter(s). Gait Posture, 21(1), 2005, p. 48-58. DOI:10.1016/j.gaitpost.2003.11.006

[8] Adkin, A.L. et al: Trunk sway measurements during stance and gait tasks in Parkinson's disease. Gait Posture, 22(3), 2005, p. 240-249. DOI:10.1016/j.gaitpost.2004.09.009

[9] Corinne, G.C. et al: Influence of virtual reality on postural stability during movements of quiet stance. Neurosci. Lett., 451(3), 2009, p. 227-231. DOI:10.1016/j.neulet.2008.12.057

[10] Cakrt, O. et al: Balance rehabilitation therapy by tongue electrotactile biofeedback in patients with degenerative cerebellar disease. NeuroRehabilitation, 31(4), 2012, p. 429-434. DOI:10.3233/NRE-2012-00813 
[11] Hejda, J. et al: Motion Capture Camera System for Measurement of Head and Shoulders Position. Biomed. Tech., 57, 2012, p. 472-475. DOI:10.1515/bmt-2012-4123

[12] Micera, S. et al: Assessment technologies for the analysis of the efficacy of a vestibular neural prosthesis. Biomed. Tech., 10, 2010, p. 55-94. DOI:10.1515/BMT.2010.491
[13] Raya, R. et al: Wearable inertial mouse for children with physical and cognitive impairments. Sens. Actuat. A Phys., 162(2), 2010, p. 248-259. DOI:10.1016/j.sna.2010.04.019

[14] Casolo, F.: Elbow prosthesis for partial or total upper limb replacements. In: "Motion control", Rijeka: InTech, 2010, p. 315-324, ISBN 978-953-7619-55-8. DOI: $10.5772 / 6972$ 